\title{
SURFACE-MOUNTED PERMANENT MAGNET BRUSHLESS MOTOR SENSORLESS CONTROL BASED ON EMF EXTRACTION AND SIMPLE DIGITAL FILTERING
}

\author{
O. M. Arafa ${ }^{(1)}$, O. A. Mahgoub ${ }^{(2)}$, M. N. Eskander ${ }^{(3)}$ \\ ${ }^{1,3)}$ Electronics Research Institute, El-Tahrir street, Dokki, Cairo, Egypt \\ ${ }^{2)}$ Department of Electrical Power \& Machines, Cairo University Giza, Egypt
}

\begin{abstract}
On-line extraction of the rotational back EMF of surface-mounted permanent magnet brushless motors (PMBM) with a reasonable degree of accuracy offers a possibility for using simple digital filtering to realize high performance sensorless control of such motors. Such possibility is investigated theoretically and experimentally in the work reported in this paper. Although the principle is applicable to both two PMBM motor types i.e. those with sinusoidal or trapezoidal back EMF, the sinusoidal type is selected as a representative case. Both simulation and experimental results ensure the effectiveness of the proposed technique.
\end{abstract}

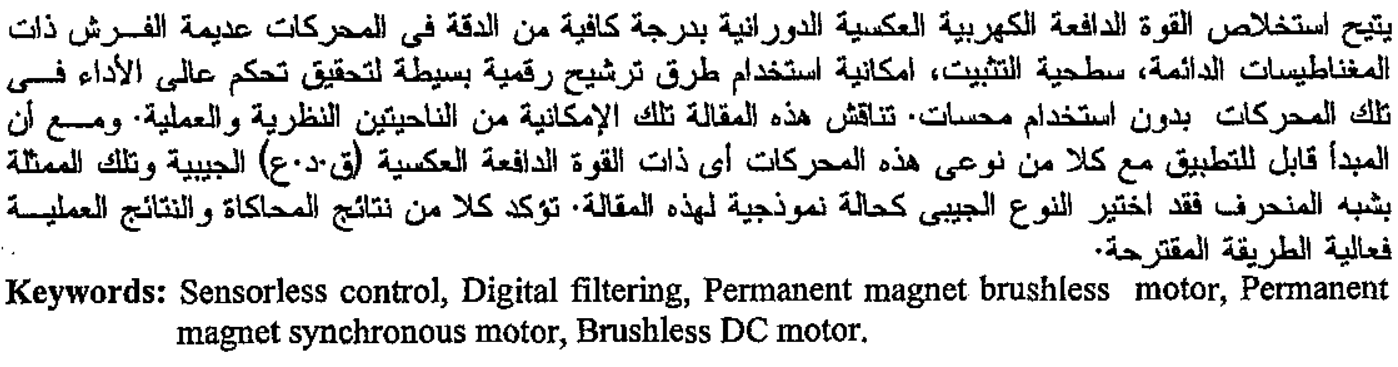

\section{INTRODUCTION}

The importance of permanent magnet brushless motor (PMBM) as a basic element in .high performance servo drive systems stems from its high efficiency, low inertia and its high torque density. Sensorless control of PMB motors, which normally operate using position and speed sensors, effectively overrides undesirable features pertaining to mechanical and/or optical sensors. This include extra cost, wiring complexity, reduced reliability, incompatibility with some harsh working environment and demand for additional mounting space [1-3].

Complex model-based filters (estimators) like extended Kalman filter (EKF) have been recently proposed and implemented for sensorless control of PMBM using the huge computational power of modern digital signal processors (DSP) [4-7] Although high-performance sensorless PMBM drives can be realized using EKF methods, such methods are characterized by intensive math manipulations and are somehow difficult to tune.

The authors of this paper have shown [7] that the independence of stator inductances on rotor position in surface-mounted PMBM makes it possible to extract the rotational back EMF (due to rotor magnets rotation) of such motors while the stator windings are under excitation.

The primary tests carried out on an experimental setup have also shown that the accuracy with which the back EMF is extracted on-line was quite reasonable and inviting for simpler digital filtering other than EKF which was proposed in [7]. Subsequent noise analysis indicated that a combination of a low pass finite impulse response filter and a median filter are quite sufficient. The filtered waveforms can be used for direct calculation of rotor position and speed.

This paper presents the details of applying simple digital filtering to the sinusoidal back EMF motor -as a representative case- along with the potential related problems and its proposed remedies. The experimental setup and the critical features of hardware implementation are highlighted. Sirnulation and experimental results are also given. The motor model and the EMF extraction method are all presented in detail in [7], so these topics will not be repeated here.

Engineering Research Journal, Vol.28, No. 2 April 2005, PP 143-150 


\section{DIGITAL FILTERING}

\section{A) FIR FILTER}

The mathematical formula describing this filter (Figure. 1) is given by the following equation:

$$
Y=\sum_{i=0}^{N} a_{i} X(n-i) \text {. }
$$

where $\mathrm{Y}$ is the current filter output, the $\mathrm{X}(\mathrm{n}-\mathrm{i})$ are current or previous filter inputs. The $a_{i}$ 's are the filter's feed forward coefficients corresponding to the filter's zeros. If this filter is excited with an impulse, the output is present for only a finite $(\mathbb{N})$ number of computational cycles. Due to its all zero structure (no poles at all), the FIR filter has a linear phase response in most standard filter applications.

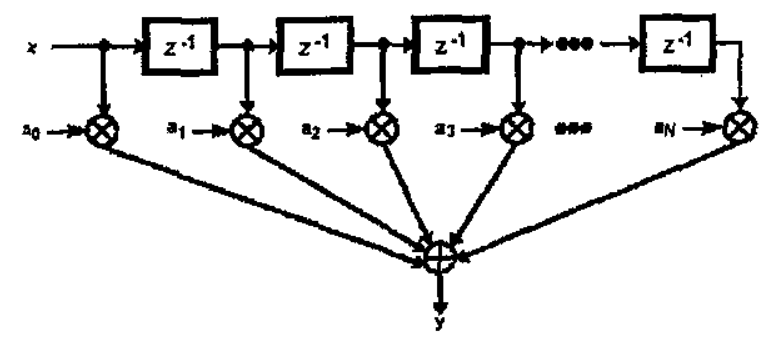

Fig. 1 FIR Filter implementation.

\section{B) MEDIAN FILTER}

For an input signal $\mathbf{V}$, the vector $\mathbf{U}$ is an $n$-element vector whose elements are taken from the elements of $\mathbf{V}$ by a window sliding over the input signal $\mathbf{V}$, where $\mathrm{n}$ is the window size of the median filter. Normally $n$ is chosen to be an odd number, then if $\mathbf{Y}$ $=\operatorname{median}(\mathbf{U})$ and the elements of $\mathbf{U}$ are rearranged in ascending or descending order in the vector $S$, the output $\boldsymbol{Y}$ is the central element of the vector $\mathbf{S}$ as follows

$Y=\mathbf{S}((n+1) / 2)$

For on-line filtering, this implies that the last $n$ samples of a noisy signal should be always kept in memory and refreshed in a first-in first-out dropping style.

\section{NOISE ANALYSIS AND PROPOSED FILTER}

In a drive system composed of a motor fed by a current-regulated voltage source inverter, noise is expected as:

- white noise due to surrounding environement.

- impulse noise due to high frequency current switching.

White noise (similar to white light) occurs with similar amplitudes over a wide frequency spectrum. Impulse noise is a momentary perturbation, limited in the frequency band, and often at saturation or maximum signal level permitted.
While finite impulse response (FIR) digital filters can be used to effectively filter white noise, impulsive noise can't be filtered by FIR without introducing large phase shift and amplitude attenuation. Median filters are more suitable to deal with impulsive noise since they introduce very little phase shift and ideally no magnitude attenuation. Also it is particularly useful in preserving the flat top of the back EMF waveform in trapezoidal motors. A combination of both types of filters is used in the next. simulation runs to produce minimum position estimation error.

\section{DIRECT EMF-BASED POSITION AND SPEED CALCULATION}

The transformation of the extracted three phase EMF from stationary frame to the two phase stationary frame results normally in a vector whose magnitude linearly represents the rotor electrical speed and whose angle represents the electrical rotor position.

\section{RELATED PROBLEMS}

Since both vector's magnitude and phase would be affected by filtering, we have got two problems:

a) A speed calculation error due to amplitude attenuation caused by the filter.

b)A position calculation error due to phase lag introduced by the filter.

Another problem arises if the drive's operational requirements involve speed-reversing. As the speed gets closer to zero, position estimation error gets higher and higher due to poor signal to noise ( $\mathrm{S} / \mathrm{N}$ ) ratio, and eventually we may loose any trustable information about the real rotor position. Therefore a modified estimation technique should be provided for position and speed in the vicinity of zero speed under speed reversal conditions. A similar situation at drive's startup is normally fixed by open loop motor acceleration.

\section{PROBLEM FIXING}

\section{A) COMPENSATION OF SPEED CALCULATION ERROR}

The first problem can be resolved by considering a hypothetical value for the permanent magnet flux coefficient. This hypothetical value is initially assumed equal to the machine EMF constant $\lambda$ upon drive startup. Then it can be tuned on line by properly differentiating the position signal over integral electrical cycles to get the actual speed under steady state conditions. This procedure effectively accounts for both the temporary change of the magnet flux coefficient due to temperature rise and the attenuation of the EMF's vector magnitude in the $\alpha-\beta$ frame due to filtering. 


\section{B) COMPENSATION OF POSITION CALCULATION ERROR}

Through the off-line simulation, the relation between phase shift introduced by any given filter combination with known parameters and speed can be predicted starting from standstill and up to the rated motor speed. It has been shown by simulation that it has a linear change profile, which makes online compensation easier. Starting from this point, we assume that the extracted back EMF vector mapped in the two phase stationary frame after applying digital filtering is $\boldsymbol{E}_{\alpha^{\prime} \beta}$. The vector $\boldsymbol{E}_{\alpha^{\prime} \beta^{\prime}}$ has a space angle $\theta^{\prime}$ with respect to the axis $\alpha^{\prime}$ of the coordinate system $\alpha^{\prime}-\beta^{c}$ as shown in Figure 2. According to the estimated speed and lookup table, $\boldsymbol{E}_{\alpha^{\prime} \beta^{\prime}}$ is lagging the actual back EMF vector of the machine $E_{\alpha \beta}$ when the latter is mapped in the same reference frame by an angle $\delta$ (obtained from lookup table).

There is some new stationary reference frame or coordinate system $\alpha-\beta$ that is not affected by the filtering process, obtained by rotating the stationary coordinate system $\alpha^{\prime}-\beta^{\boldsymbol{c}}$ against the direction of motor rotation with an angle $\delta$ (advance) such that the projections of the vector $\boldsymbol{E}_{\alpha^{\prime} \beta^{\prime}}$ on its $\alpha-\beta$ axis would give a resultant vector angle $\theta$, where $\theta=\theta^{\prime}-\delta$.

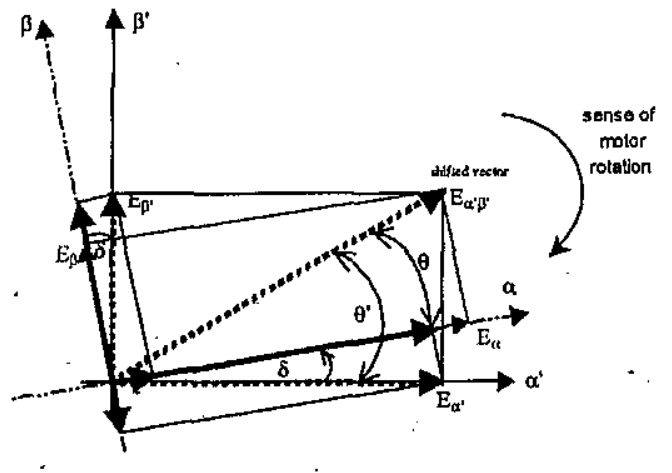

Fig. 2 Frame rotation

In this way, the phase shift introduced by the filtering process can be corrected. The projections of the vector $E_{\alpha^{\prime} \beta^{\prime}}$ on the axes of the new coordinate system can be deduced from the geometry of Figure 2, and they are given by equation (10).

$$
\left[\begin{array}{c}
E_{\alpha} \\
E_{\beta}
\end{array}\right]=\left[\begin{array}{cc}
\cos \delta & \sin \delta \\
-\sin \delta & \cos \delta
\end{array}\right]\left[\begin{array}{c}
E_{\alpha^{\prime}} \\
E_{\beta^{\prime}}
\end{array}\right]
$$

\section{C) ESTIMATION AT SPEED'S ZERO- CROSSING}

Fortunately, the motor speed changes linearly with time -under almost all loading conditions- in the vicinity of zero speed during a speed reversing process. The rate of change can be captured on-line; extrapolation can be used to predict the motor-speed once the estimated speed drops under a predefined threshold until it develops above that threshold in the reverse direction. The rotor position can be estimated by simple integration process (e.g. trapezoidal rule) applied on the predicted speed within this period.

\section{SIMULATION RESULTS}

Two extremities regarding noise level are simulated and the results are shown by Figure 3-a through Figure 3-e. The first plot of each figure shows the case of extremely low-level noise while the case of extremely. high-level noise is shown by the second plot. The impact of higher noise level is generally degrading the performance. At the neighborhood of zero speed under speed reversal conditions, the alternative estimation technique is used.

It is generally noticed that the higher the noise level, the higher the estimation error. However, the error limits reached are well tolerable. Simulation results for the case of extremely high noise level are presented as a boundary case, under practical conditions much better performance have been already obtained since noise level is much lower than what we have used in simulation.

\section{EXPERIMENTAL RESULTS}

An experimental setup was built using a 400 watt motor whose parameters are given in the appendix. The exact synchronization of the readings of currents and voltages which is necessary for accurate EMF extraction [7] is realized by building a customdesigned data acquisition board.
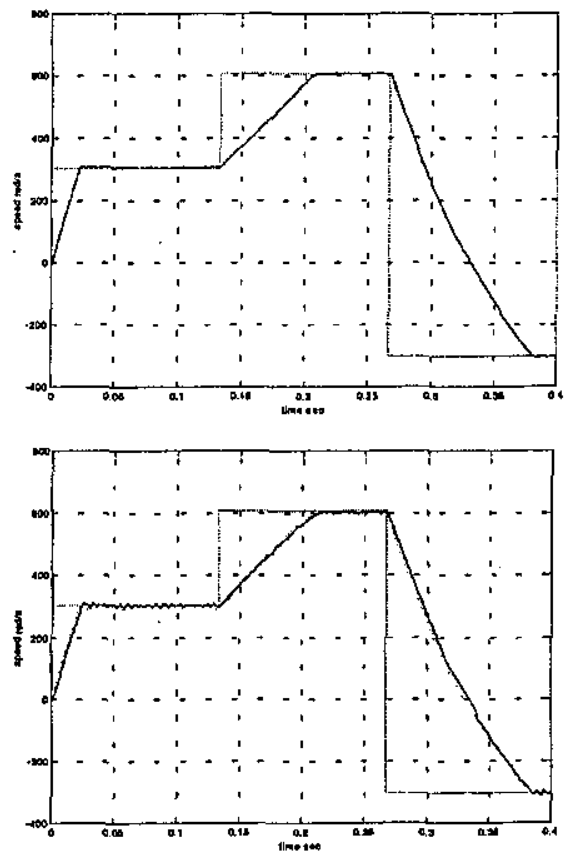

a) Motor Speed Profile 

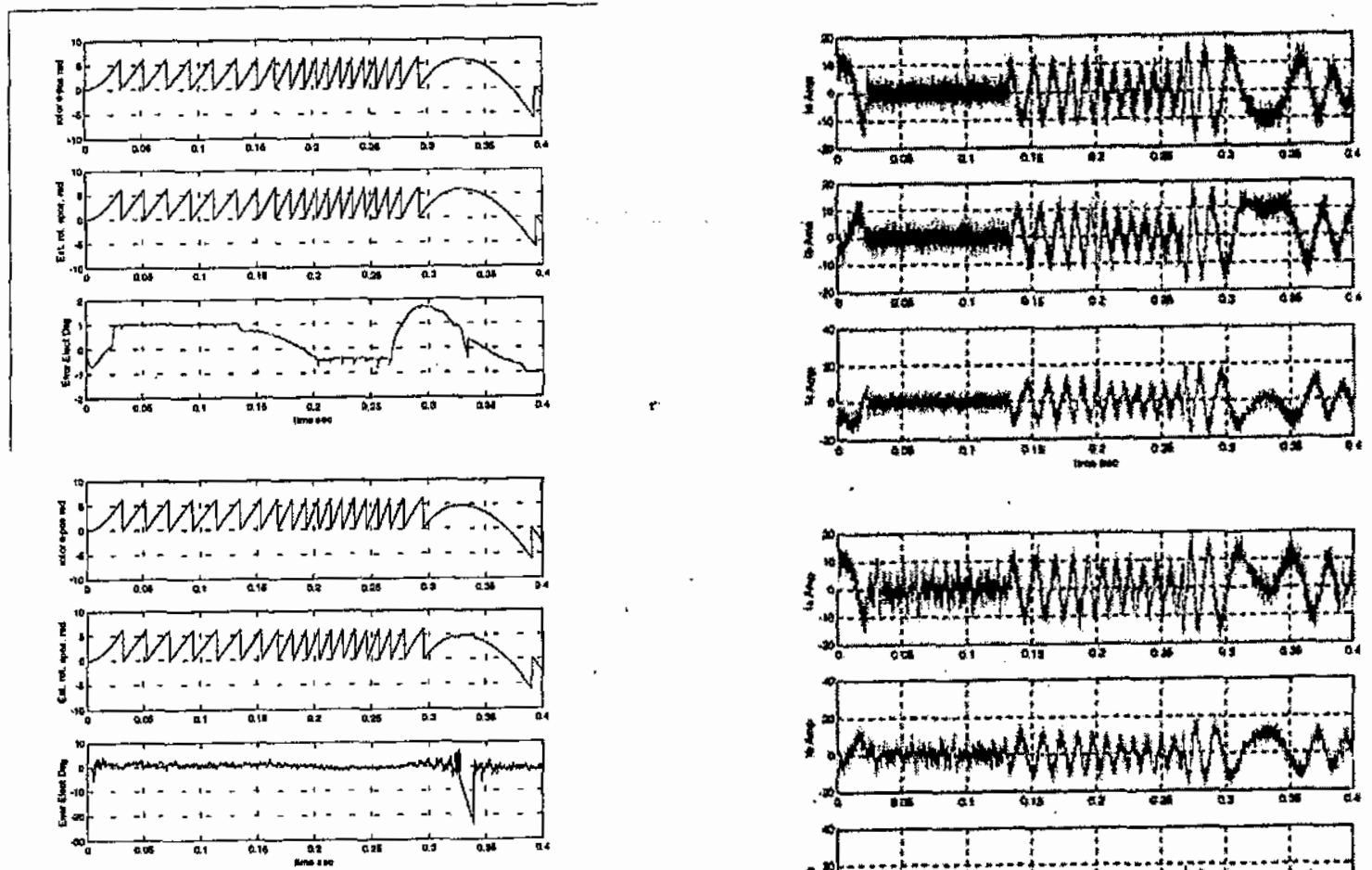

b) Electrical Rotor Position (Actual/Estimated/Error)
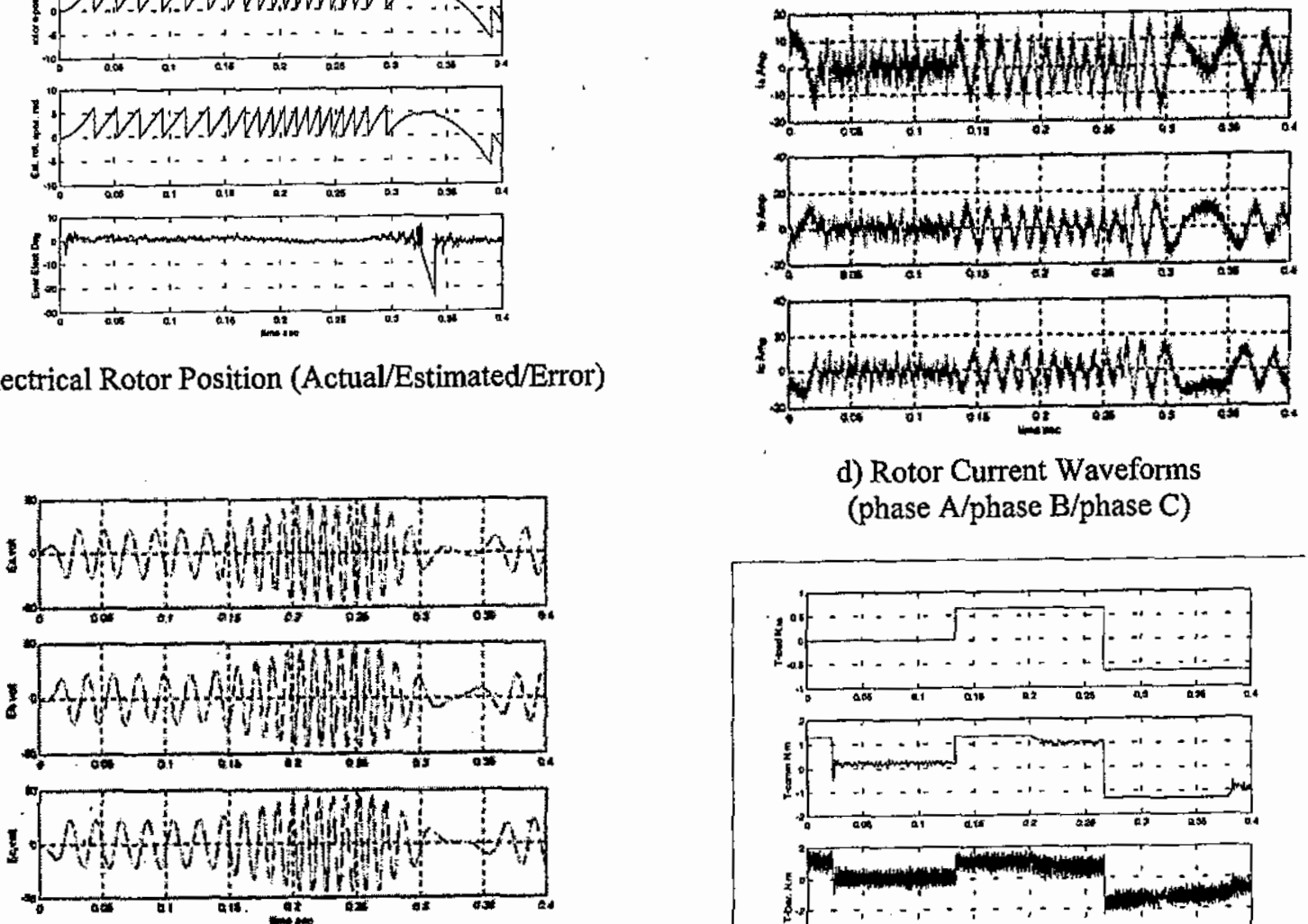

d) Rotor Current Waveforms (phase $\mathrm{A} /$ phase $\mathrm{B} /$ phase $\mathrm{C}$ )
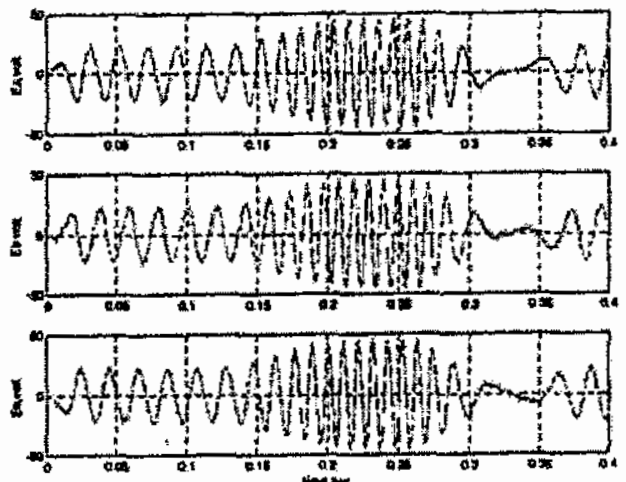

c) EMF Extracted by the Proposed Method (phase A/phase B/phase C)
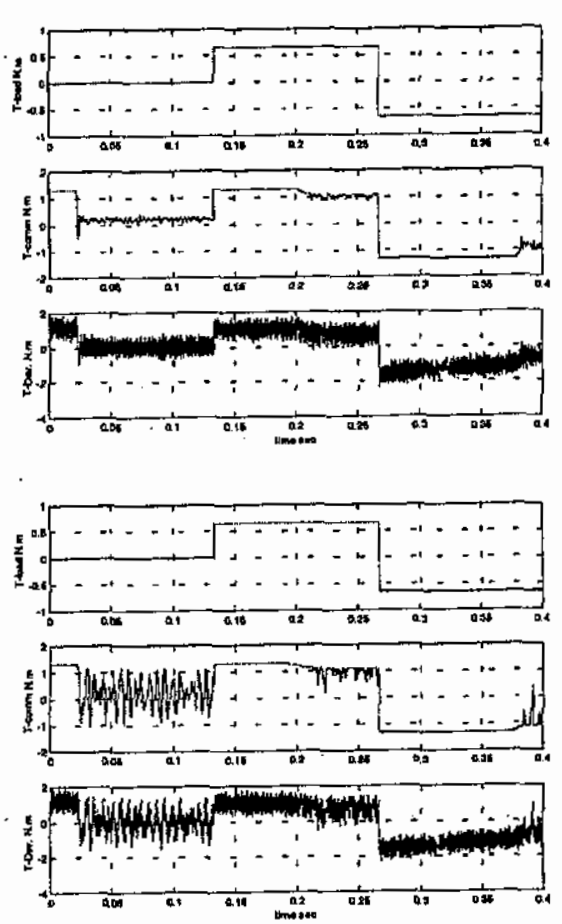

e) Motor Torque Profile

(Load/Command/Developed)

Fig. 3 PMSM performance curves with sinusoidaltype excitation 
This board is equipped with sufficient number of simultaneous sampling and converting $\mathrm{A} / \mathrm{D}$ channels. The board is also equipped with $\mathrm{D} / \mathrm{A}$ channels, digital input/output lines and timing resources. The board is shown in Figure 4 and it is hosted in an ISAbus of a PENTIUM II $333 \mathrm{MHz}$ personal computer.

Position and speed measurements are made for comparison with estimated quantities via an incremental encoder integrated with the PMBM motor.

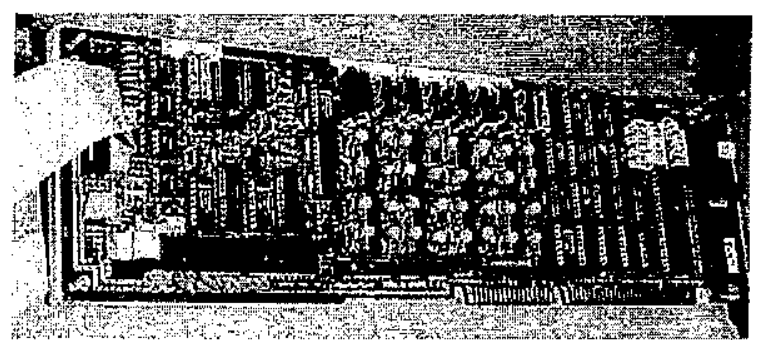

Fig. 4 Custom-built data acquisition board

The control program is coded in $\mathrm{C}++$ language. Center-aligned space vector pulse width modulation (SVPWM) is executed at a frequency of about 10 $\mathrm{KHz}$ by the $\mathrm{PC}$ processor using time-controlled interrupts.

Analog measurements are synchronized with the center line of each PWM period by means of software. Motor loading is realized by coupling a separately excited DC generator feeding a suitable resistive load as shown in the setup overview photograph of Figure 5.

\section{ON-LINE EMF DETECTION}

On-line extraction of the back EMF is verified through oscilloscope traces in this section. In Figure 6 the upper trace shows the differential voltage measurement across the external inductor connected to phase " $\mathrm{A}$ ". The high frequency voltage component is due to the inductor reaction with the fast switching process of the inverter, while the lower frequency voltage changes is due to the inductor reaction with the fundamental component of the winding current. The second trace shows the terminal voltage measured at the motor terminal of phase A.

The trace resembles portions broken out of a sinusoidal wave and impaired by some offset and high frequency noise. The third trace shows the extracted EMF resulting from applying the algebraic extraction formula given in [7], the improvement established in the waveform is evident since it gets closer to a sinusoid without introducing any phase shift or magnitude attenuation. The fourth trace shows the EMF resulting after applying simple low pass FIR and median digital filtering, it is clear that some phase shift is now introduced due to this filtering. The filter's math is executed on line upon acquiring each new sample. Figure 7 gives a closer view on the time axis of Figure 6. Figure 8 further illustrates phase lag due to additional filtering.

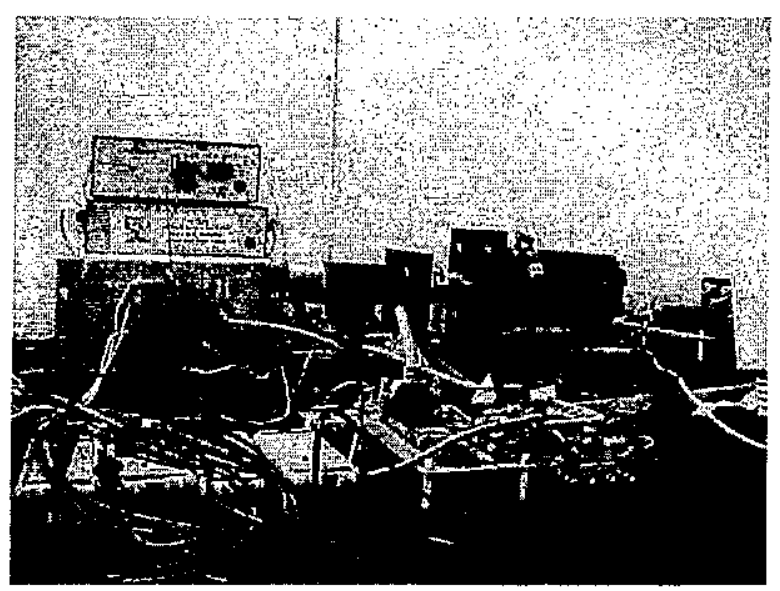

Fig. 5 Experimental setup overview

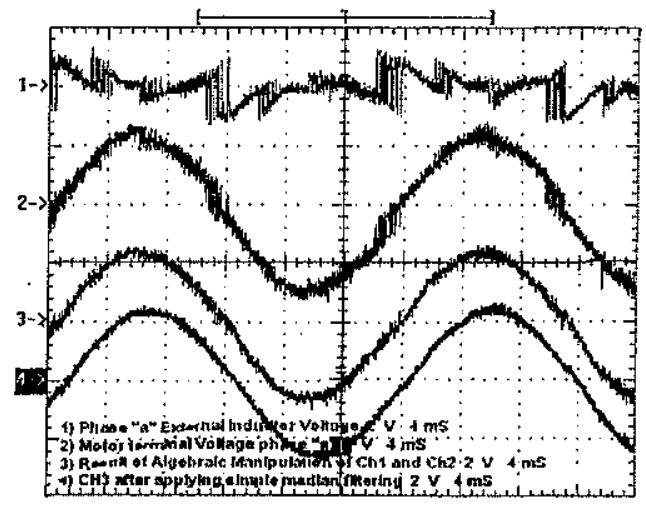

Fig. 6 Voltage waveforms / phase "A"

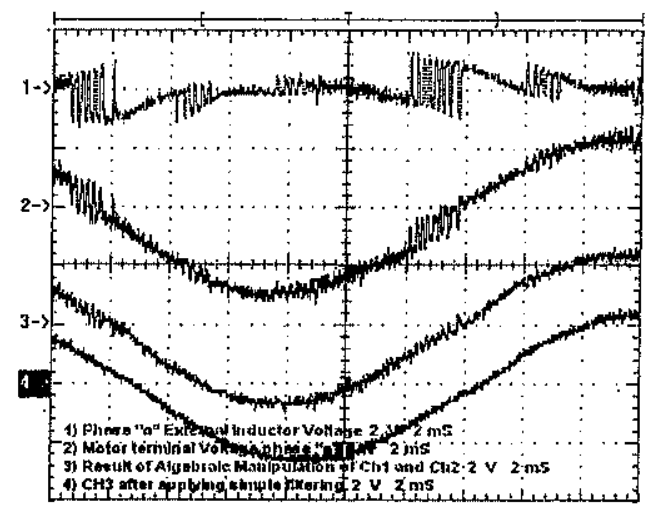

Fig.7 A closer view of Fig.(7).

Consequently, this phase lag is reproduced in the estimated position as shown in Figure 9, where the leading trace represents the measured position, the 
lagging trace represents the position calculated from the filtered EMF and the upper trace represents the algebraic difference between them. The apparently high error pulses near the $2 \pi$ transitions actually have a low value (by dropping $2 \pi$ representing the complete cycle).

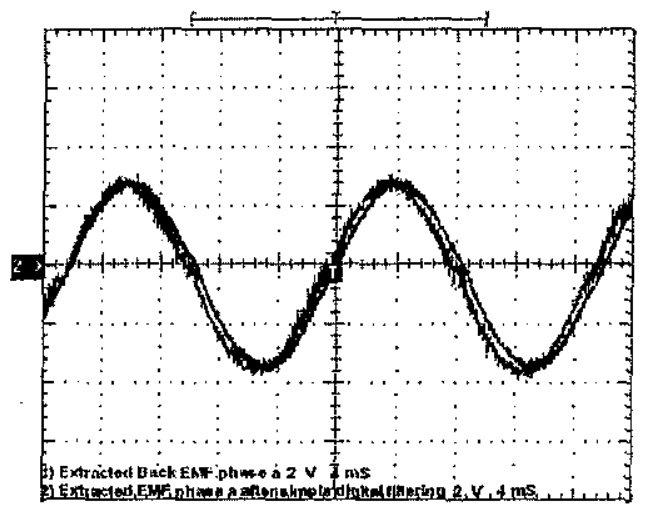

Fig. 8 Phase shift added by subsequent digital filtering

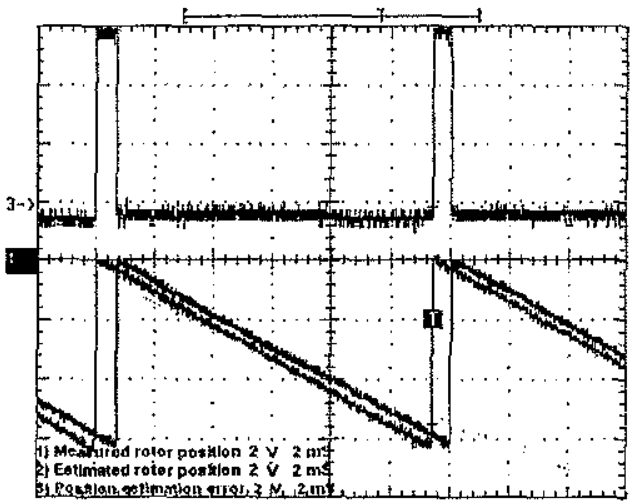

Fig.9 Phase lag due to filtering

\section{OPEN LOOP ACCELERATION}

Although the proposed technique is capable of starting the drive in sensorless mode from zero speed, the resulting sense of rotation may conform to the commanded one or not, based on the initial rotor position which is random and unknown.

Therefore, the first step in any running session is to provide an open loop acceleration period to serve two purposes:

- Guiding the direction of rotation to ensure conformance with the commanded one.

- Reaching a minimum speed to make the EMF detection more accurate and hence trustable.

Figure 10 illustrates the time profile of the open loop acceleration through the rotor position information, the trace with the inflexion point shows the actual rotor position, while the other trace shows the rotor position considered within the open loop acceleration routine which is used to provide the excitation voltage to the motor. When the acceleration rate is well tuned to the motor and load inertia, the error between the assumed position and the actual position decreases and the expected transient due to switching from open loop to closed loop sensorless acceleration is minimized and becomes more tolerable.

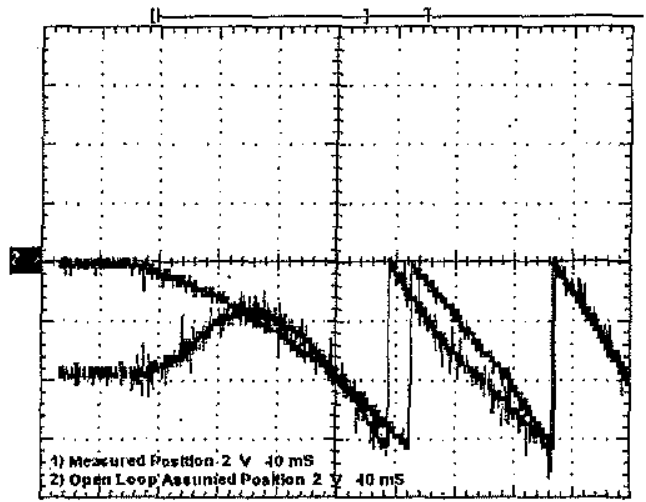

Fig. 10 Open loop acceleration, first cycles

\section{NORMAL SENSORLESS OPERATION}

Once the motor acquires a reasonable speed value, the calculated speed and position can be used directly for feeding the current controller and for closing the speed control loop. Figure 11 gives a comparative vision of the actual (measured) rotor position (first trace) and the calculated one (second trace) under motor loading conditions when the motor is running in sensorless closed loop control mode with phase shift compensator enabled. The high frequency ripples superimposed on the upper trace is merely a picked up noise.

The third trace represents the error between the actual and the estimated position. The picked up noise is reproduced in the error signal accordingly, the coincidence between the two signals are otherwise excellent.

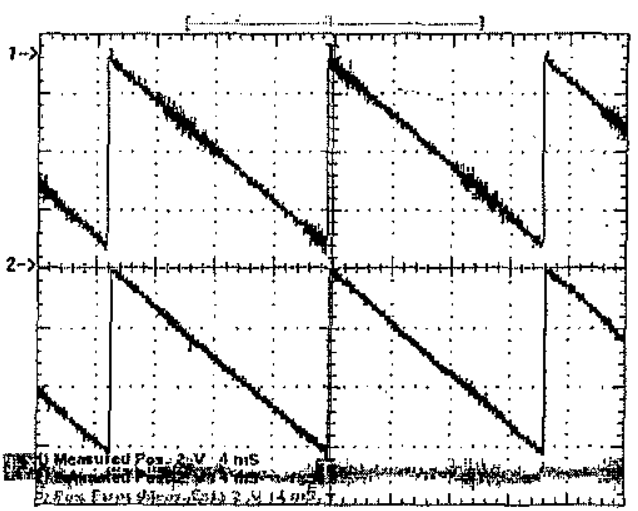

Fig. 11 Rotor position during normal sensorless operation

Figure 12 illustrates the speed step response under loading conditions, channel No.1 represents the 
speed command, and channel No.2 represents the measured speed response. The open loop acceleration is clear at the early beginning where the motor builds up some speed, during which the estimated position gets more trusted and the sense of rotation is defined (clockwise rotation is selected here).

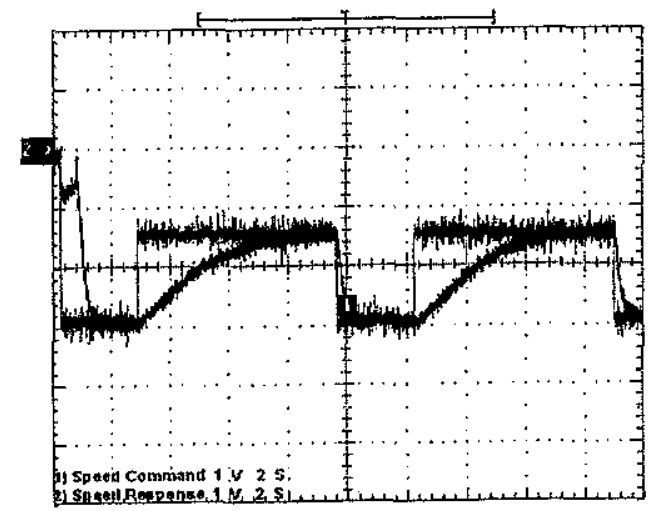

Fig. 12 Speed step-response

Control is then transferred to closed loop mode, first the speed reference was step changed from zero to $59.6 \%$ of rated speed. Then a periodic change of speed reference from $59.27 \%$ to $24.6 \%$ of rated speed and from the lower to the higher again is enforced. The motor takes longer to decelerate to the lower speed reference because it is left for free retardation during this period. Figure 13 illustrates the dynamic reaction of the three-stator currents due to the step increase in speed reference.

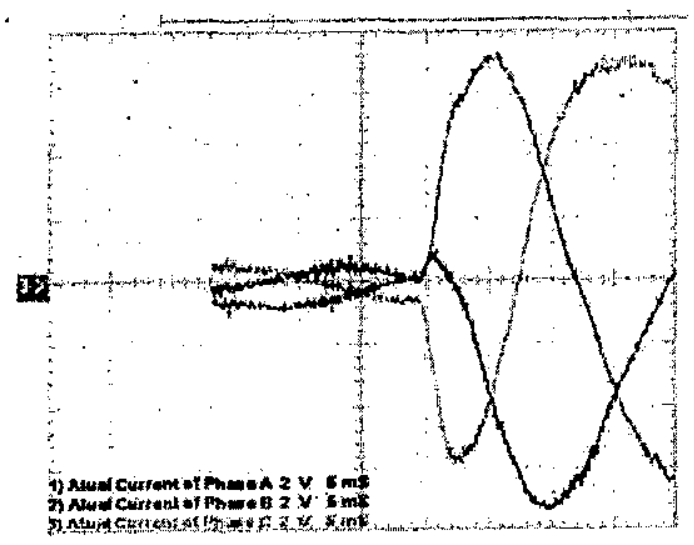

Fig. 13 Three-phase stator current profile in transient

\section{CONCLUSION}

High performance PMBM drives can be realized by effective extraction of rotational back EMF and subsequent simple digital filtering. This generally yields a low-cost solution without sacrificing the performance, so that a suitable cheap microcontroller can provide the high performance otherwise provided by expensive DSP and complicated sensorless algorithms.

A noticeable drawback is the extra voltage amount that should be supplied by the inverter to compensate for voltage drop across the external inductors; selecting inductors of proper values can minimize this drawback. Very small inductor value would result in a low measurement accuracy due to poor signal to noise ratio. A compromise between reducing the additional voltage and measurement accuracy improvement is best deciding the external inductor value. In simulation a value of $10 \%$ of motor equivalent inductance per phase for external inductor was used. The on-line computational power requirement can be satisfied by a suitable cheap microcontroller solution.

\section{REFERENCES}

[1] Ogasawara S., Akagi H., "An Approach to Position Sensorless Drive for Brushless DC Motor", IEEE Transactions on Industry Applications, September /October, 1991, PP 928-933.

[2] Kinichi Ilzuka, Hideo Uzuhashi, Minoru Kano, Tsunehiro Endo, 'Microcomputer Control for Sensorless Brushless Motor", IEEE Transactions on Industry Applications, Vol. IA21, No. 3, May/June 1985, PP 595-601.

[3] L. Cardoletti, A. Cassat, M. Jufer, "Sensorless Position and Speed Control of a Brushless DC Motor from Start-up to Nominal Speed" ,EPE Journal, Vol.2, No.1 (1990), PP 25-34.

[4] Paul Kettle, Aengus Murray, Finbarr Moynihan, "Sensorless Control of a Brushless DC Motor using an Extended Kalman Estimator", PCIM'98 Intelligent Motion. Nurenberg, May 1998, Proceedings, PP 385-392 .

[5] Petre Minciunescu, Tom Flint, Finbarr Moynihan, Paul Kettle, "Sensorless Control of Brushless DC Motors Using Extended Kalman Filter Estimator and Back EMF Integration Algorithm: A Comparison", Embedded Control Systems, Analog Devices Inc.

[6] Silverio Blognanai, Mauro Zigliotto, Marco Zordan, "Extended-Range PMSM Sensorless Speed Drive Based on Stochastic Filtering", IEEE Transactions on Power Electronics, Vol.16, No.1, Jan 2001, PP 110-116.

[7] O. M. Arafa, O. A. Mahgoub, M. N. Eskander, "Surface-Mounted PMBM Sensorless Control Based on EMF Extraction and Extended Kalman Estimator", Electrical Researches Journal, Jan 2005, N0.578, pp. 
O. M. Arafa, O. A. Mahgoub, M. N. Eskander, "Surface-Mounted Permanent Magnet Brushless

\section{APPENDIX}

The parameters of a 400-watt SM PMBM motor used in simulation are listed below:

Back EME type Sinusoidal

$\mathrm{R}$ (Resistance per phase) $=0.560$

$L^{\prime}=\mathrm{L}_{\mathrm{s}}-\mathrm{L}_{\mathrm{M}} \quad=5.945 \mathrm{e}-4$

$\lambda_{m}^{\prime}$ (Back EMF const) $=0.073$
$\mathrm{J}$ (Inertia)

$P$ (Number of poles)

Rated continuous torque

Rated Speed

Rated power

Rated Current
$\mathrm{Kgm}^{2}$

N.m.s/rad

$=2$ poles

$=660 \mathrm{e}-3 \quad \mathrm{~N} \cdot \mathrm{m}$

$=5800 \quad$ R.P.M.

$=400 \mathrm{~W}$

$=4.51 \mathrm{~A}$ 